Stocka Joanna, Simińska Joanna, Bukowska Weronika, Porzych Piotr, Ogurkowski Karol, Nowacka Krystyna, Hagner Wojciech. Rating nurses' knowledge of basic steps of cardiopulmonary resuscitation. Pedagogy and Psychology of Sport. 2018;4(2):11-19. elSSN 2450-6605.

\title{
Original text
}

Stocka Joanna, Simińska Joanna, Bukowska Weronika, Porzych Piotr, Ogurkowski Karol, Nowacka Krystyna, Hagner Wojciech. Rating nurses' knowledge of basic steps of cardiopulmonary resuscitation. Journal of Education, Health and Sport. 2018;8(2):11-19. eISSN 2391-8306. DOI http://dx.doi.org/10.5281/zenodo.1163402

http://ojs.ukw.edu.pl/index.php/johs/article/view/5239

$$
\begin{aligned}
& \text { The journal has had } 7 \text { points in Ministry of Science and Higher Education parametric evaluation. Part B item } 1223 \text { (26.01.2017). } \\
& 1223 \text { Journal of Education, Health and Sport eISSN 2391-8306 } 7 \\
& \begin{array}{c}
\text { OThe Authors 2018; } \\
\text { This article is published with open access at Licensee Open Journal Systems of Kazimierz Wielki University in Bydgoszcz, Polan }
\end{array} \\
& \text { Open Access. This article is distributed under the terms of the Creative Commons Attribution Noncommercial License which permits any noncommercial use, distribution, and reproduction in any medium, } \\
& \begin{array}{l}
\text { provided the original author(s) and source are credited. This is an open access article licensed under the terms of the Creative Commons Attribution Non Commercial License } \\
\text { (http://creativecommons.org/licenses/by-nc/4.0/ which permits unrestricted, non commercial use, distribution and reproduction in any medium, provided the work is properly cited. }
\end{array}
\end{aligned}
$$




\title{
Rating nurses' knowledge of basic steps of cardiopulmonary resuscitation
}

\author{
Stocka Joanna, Simińska Joanna, Bukowska Weronika, Porzych Piotr, \\ Ogurkowski Karol, Nowacka Krystyna, Hagner Wojciech
}

Department of Rehabilitation, Medical College in Bydgoszcz, Nicolaus Copernicus University in Torun

\begin{abstract}
First aid is provided by witnesses of the event, the Therefore it is important That Provide every citizen could this assistance in a correct and effective manner until the arrival of the medical rescue team. From the witnesses of the event, the chances of survival of the survivor's or his health detriment that are largely dependent. Education in the field of first aid starts already in primary school, however, it is important That the students of this class can use the knowledge in practice
\end{abstract}

Key words: first aid, premedical help, resuscitation

Granting medical aid is the responsibility of every adult citizen talks about the Penal Code and the Law on State of Emergency Medicine 2006 and the Road Traffic Act. No first aid may lead to imprisonment up to three years. The exceptions are situations where first aid could be life-threatening rescuer. Therefore it is important that every adult citizen has a basic knowledge of first aid. The rationale for a knowledge of first aid is the fact that between the event and the arrival of the emergency services usually takes several minutes that are essential for life and health of the injured person. Recommended arrival time for the emergency medical team should be no longer than 8 minutes often due to various external factors, ie time. distance from the scene of the incident, the current traffic can be extended. Proper brain function without a supply of oxygenated blood is able to maintain for 3-5 minutes. This shows the need for first aid by bystanders who They are highly likely to contribute to the survival of the victim. In recent years there has been increased emphasis on the responsibility and effectiveness of 
CPR undertaken by witnesses. The efficiency of these operations is very important for the survival of patients with cardiac arrest $[2,16]$.

\section{Resuscitation and reanimation}

Resuscitation is to restore the patient from death or potential sham, Death by clinicians is defined as the cessation of cardiac respiratory function and brain function. Factor influencing the behavior of resuscitation is to maintain the flow of oxygenated blood to the heart and brain. $[1,4,7]$

Resuscitation CPR differs from that marks the return of the vital functions together back to the patient's awareness [7,17].

Sudden cardiac arrest

Cardiac arrest defined as a state in which there is a sudden stop of cardiac function and the operation of the cardiovascular system. Leading next to stop the function of the respiratory system and, consequently, to the termination functions of the central nervous system [3].

An acute threat to life is defined as the deterioration of health suddenly or envisaged in the near future, which can lead to damage to the usual functions of the organism or life [9].

Sudden cardiac arrest is a condition of having a critical heaviest symptoms and the most common among medical cases. The most common cause of sudden cardiac arrest are cardiac disorders [11]

Among the causes of sudden cardiac arrest can be distinguished: [10]

- the most common: acute coronary syndrome,

- cardiomyopathy,

- genetic heart disease,

- valvular heart disease,

- Heart arythmia,

- multi-organ injuries,

- poisoning,

- water and electrolyte disturbances

The symptoms of sudden cardiac arrest include [19];

- loss of consciousness,

- no pulse on large arteries, 
- abnormal breathing or apnea

Cardiac arrest can occur in ventricular fibrillation or pulseless ventricular tachycardia, asystole, and pulseless electrical activity. Electrocardiogram chaotic uneven line at a frequency of 250-500 / min with a different amplitude and absence of gaps between the QRS wave band is described as fibrillation. Lack of adequate medical treatment for this type of disorder leads to death within a few minutes.

Another threat is the disorder which asystole means no electrical activity of both atria and ventricles. This type of cardiac arrest preferably does not affect the prognosis of the patient. $[5,6]$

Basic life support

Action witnesses is an essential element of the "chain of survival". Early initiated correct cardiopulmonary resuscitation significantly increases the chances of saving the life of a person with cardiac arrest. Basic life support (BLS) are taken after an assessment of the victim by means of an algorithm. A proper resuscitation consists of several steps according to a predetermined algorithm $[8,13]$.

BLS in adults

Before the approach to the injured person who takes salvage operations should check the safety of the scene, if the thread does not threaten the victim and the person taking up the first aid should go to perform rescue operations.

Check the reaction of the victim gently shaking his arm and saved or responds to voice,

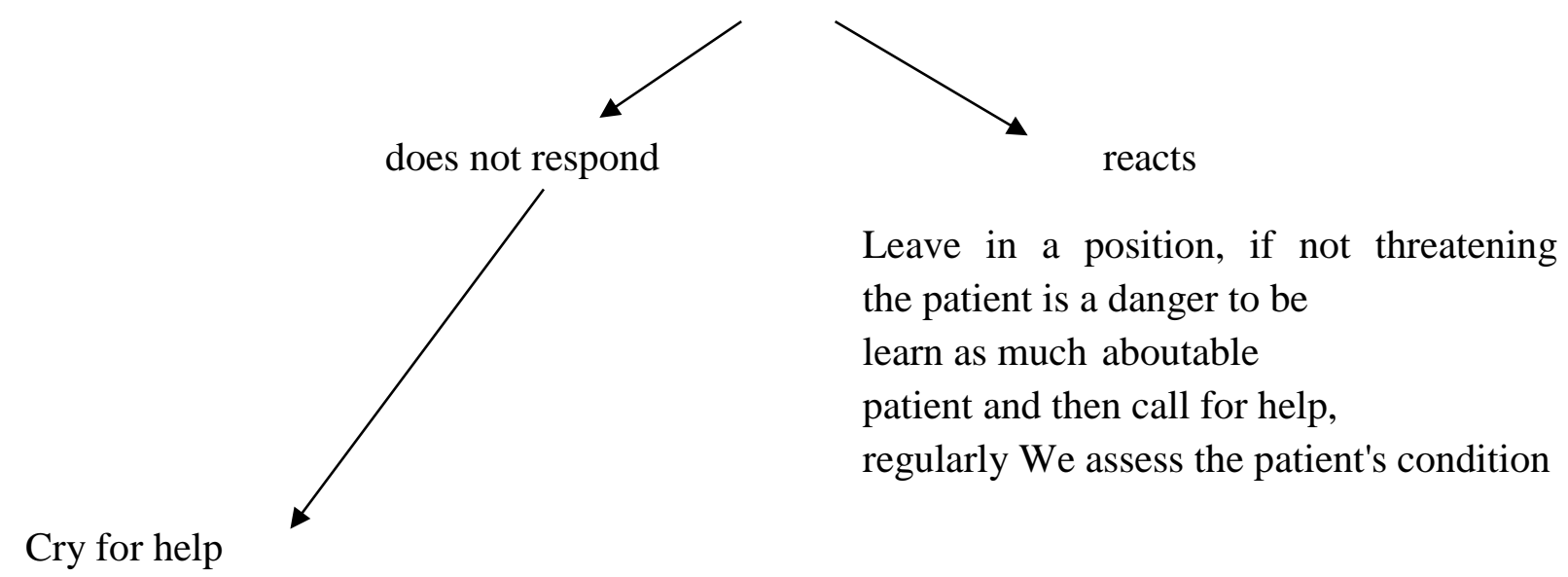

It should be loud call for help, to turn the victim on his back, 


\section{Open airway inhalation}

by head tilt and chin lift, keeping the airway: sight, hearing and touch judge breath, or chest rises, sense the movement of air in their breathing policzku- should assess whether it is correct, incorrect or absent, the assessment breath allocated to 10 seconds

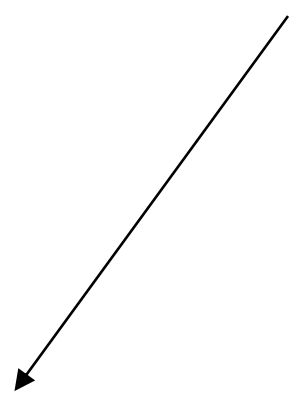

Not breathing

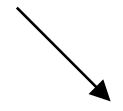

breathing normally

victim placed in position safe, call help-112 or 999 regularly

We evaluate the patient's breath,

call 112

$\downarrow$

30 chest compressions

2 breaths $[14,15]$

BLS in children

Same as in adults before making rescue work safety assessment at the scene.

Check the state of consciousness of the child gently shake the child's shoulder and see if the child reacts to the voice

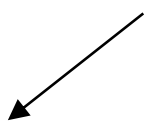

does not respond

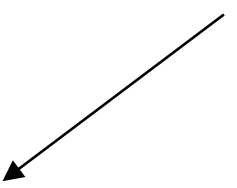

Cry for help

join resuscitation

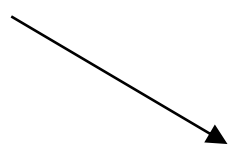

reacts

if the child should answer us innikliwie observe the patient's condition 
lifting sunken root of the tongue, look, listen and feel for 10 seconds breathing evaluate the presence or lack thereof,
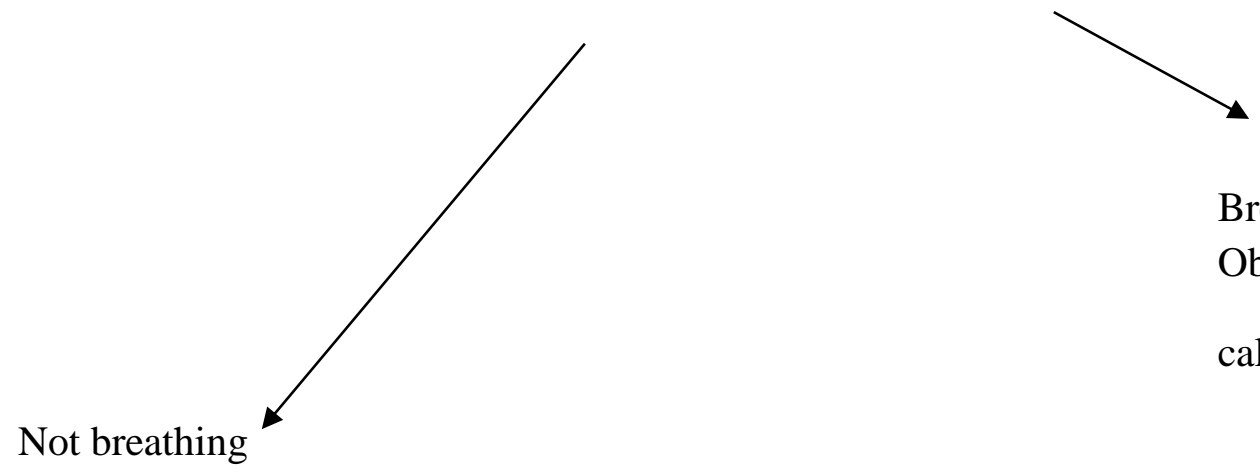

Breathable

Observation

call 112

call 112

If we find no breathing or if breathing is irregular or inefficient should seek potential foreign bodies in the mouth of the child,

5 rescue breaths

15 compressions

Two rescue bleaths

\section{Education in first aid}

Education is education, education, all activities and processes aimed at the transfer of knowledge, development of specific qualities and skills.

Education in first aid is extremely important because in the event can be witnessed every one of us and then the algorithm knowledge of first aid is extremely valuable.

The aim of the study is to compare the knowledge of first aid among students, medical personnel and firefighters how

A study of nurses in Kujawsko Pomorskie by the author of an anonymous questionnaire. It filled in a questionnaire of 70 nurses and 30 nurses aged between 20 and 60 years working in departments of Anesthesiology and Intensive Care, Pediatric Hospital and emergency department. The survey contained 100 questions where the maximum number of points available was 22. 52\%. Respondents showed a high knowledge of first aid, $21 \%$ of respondents received less than 16 points which meant the average level of knowledge first aid. The low level of knowledge referred to the people who earned less than 11 points, they accounted for $27 \%$. Education of respondents have an impact on the state of knowledge of first aid, people with post-secondary education in more than half showed a low level of knowledge while those with undergraduate education 
and MA exhibited a high state of knowledge. The results showed no relationship between gender and knowledge of first aid. It did demonstrate a relationship between the age and knowledge of the surveyed BLS where on average $86 \%$ above 60 years of age showed a low level of knowledge, and $78 \%$ of nurses between 20 and 27 years of age were characterized by a high state of knowledge in this field. Branch the subjects in which they worked had an impact on their state of knowledge $45 \%$ of people working in the emergency department had a low level of knowledge, while colleagues from OIOMU 94\% showed a high level of knowledge. [7]

In Wielkopolskie study was conducted on 117 students of 72 IV and 82 of the medical interns doctors. As a form of a questionnaire survey were selected multiple choice knowledge checking the basic steps of cardiopulmonary resuscitation - breathing in adults, children and infants. The analysis concerned such issues as the ratio of compressions to breaths in a sevenyear, pending the evaluation of pulse sequence resuscitation techniques compressions in infants. Most errors committed in the questionnaire firefighters least doctors. Medicom most difficult question of the place made compressions in infants. Firefighters had the biggest problem

identifying the sequence of operations with cardiopulmonary resuscitation breathing. Medical students as well as doctors had a problem with specifying the place of oppression cage a baby's. $13 \%$ of physicians surveyed answered correctly on all issues. Among the students and firefighters there was no survey dissolved in $100 \%$ improved. Firefighters and students respectively 13.7 and $12.5 \%$ did not answer any question correctly. The survey $86 \%$ of the respondents did not have the knowledge base first aid [2].

Another study was to demonstrate the students' knowledge of Poznan and to show possible changes in the transfer of knowledge of first aid. Tested 393 students at the Poznan University of Technology (74 people), Medical University (107 people) and the University of Economics (144 persons) and the university. Adam Miciewicza (68 people). Were assessed evaluation of knowledge and willingness to take action and rescue own knowledge in this field. Women accounted for $64.6 \%$ of men and $35.4 \%$ of the group. The age range of respondents are between 19-26 years, only 1\% of the respondents had more than 26 years. In the first part, students evaluated the Polish society preparation for first aid, $70 \%$ of respondents said that Poles are not well enough trained to save lives. When it comes to self-esteem in first aid it was identical to the profile of the university and that $68 \%$ of students at the Medical University evaluated their knowledge as good or very good, Collision with students of the University of Economics in only $19 \%$ have identified their knowledge as a good while because more than half of $69 \%$ defines it as insufficient. Similar results were obtained at the University. Adam Mickiewicz where 59\% considered their knowledge as insufficient. Both students from the Adam Mickiewicz University and the University of Economics in any percentage of their knowledge do not pointed at a very good level. Another source of knowledge rebuked analyzed with first aid. Students of the University Medical encountered $91 \%$ Another source of knowledge rebuked analyzed with first aid. Students of the University Medical encountered 91\% Another source of knowledge rebuked analyzed 
with first aid. Students of the University Medical encountered 91\% with the principles of first aid during classes. The second place where the subjects had contact with first aid classes of high school and a technical college. For other respondents university as a place of contact with classes in first aid they pointed ponaddgimnazjalne school. As other places to gain knowledge, respondents indicated driving courses, scouting, rescue WPOR courses, sailing and powerboat courses and courses to prepare for camp counselors. Willingness to participate in the first aid course was declared by more than $50 \%$ of respondents to refresh knowledge. Other questions concerned knowledge of first aid and outside the Medical University students other students had insufficient knowledge. The main source of knowledge of first aid among college students is not medical and upper secondary school for students of the Medical University classes in the course of study. The vast majority of medical students know the algorithm proper conduct among the non-medical fields only every other student is able to identify the correct sequence [18].

\section{Summary}

The medical profession in May a much better knowledge of First Aid ranges than the rest of society. This may result from the permanent contact and monitoring of the patient's health status and knowledge of current issues arising from the profession. People with medical industry last contact with classes in first aid May in the course of study which influences: the freshness of knowledge, while the remaining respondents indicate profession last place in contact with first aid classes at the school average. Of great importance is also studied education where a better knowledge in the field of rescue are people with higher education than those with post-secondary. Observing the above comparison it must be concluded that more emphasis should be placed on education and teaching procedures, first aid because under the stress of some life saving actions should be automatic to the uncomfortable conditions do not make a mistake and thus does not reduce the effectiveness of first aid.

\section{Bibliography}

1. J. Andres, First aid and cardiopulmonary resuscitation. Polish Resuscitation Council. Kraków 2006,

2. Dabrowski M. Dabrowska A. Sip M. Witt M. Investigation of knowledge of basic issues in resuscitation rescuers PSP, medical students and doctors in training, Medical News 2012, 81, 6,

3. Hryniewiecki T., Emergencies edition 3. Medical Tribune Poland. Warsaw 2014

4. Jakubaszko J. Emergency Medicine. The sudden threat of internal origin. Górnicki medical publisher. Wroclaw 2003

5. Karpinski G., M. Grabowski, Filipiak KJ Opolski G., Acute coronary syndromes, electrocardiographic atlas. Medical publisher Urban \& Partner. Wroclaw 2004

6. Krajewska-Kulak E., M. Szczepanski, physical examination in the practice of nurses and midwives. Publisher helm. Lublin 2008, 
7. Krysiak E. Evaluation of knowledge of resuscitation and cardiopulmonary resuscitation nurses, hospital emergency department and clinical departments. Wloclawek 2016

8. For help. Magazine for life-saving services. Publisher Elamed. Katowice 4/2010 Year 4 (XVI)

9. At the Rescue, Magazine for life-saving services. Publisher Elamed. Katowice 3/2010 Year 4 (XV)

10. Rescue Magazine for life-saving services. Publisher Elamed. Katowice Year 2/2010 4]

11. At the Rescue, Magazine for life-saving services. Publisher Elamed. Katowice I 2010 Year 4 (XIII)

12. To the rescue, the magazine for life-saving services. Publisher Elamed. Katowice 42009 Year 3) XII

13. For help. Magazine for life-saving services. Publisher Elamed. Katowice 2/2008 Year 2 (VI)

14. For help. Magazine for life-saving services. Publisher Elamed. Katowice I / 2008 Year 2 (V), p. 32-35

15. For help. Magazine for life-saving services. Publisher Elamed. Katowice 4/2007, pp. $50-52$

16. D. Olejniczak Miciuk Religioni D. Laws Assessment of Knowledge students of the Medical University majoring in nursing about first aid, Piel. Zdr. Publ. 2013, 3, 2,

17. Plantz S., Wipfler E. Emergency Medicine. Urban \& Partner. Wroclaw 2007

18. Skitek I. M. Witt Goniewicz M. Evaluation of knowledge of first aid issues among students of Poznań University, Medical News 2012, 81, 6

19. Szczeklik A., P. Gajewski Internal Medicine compendium practical medicine. Publisher practical medicine. Kraków 2010, pp. 106-108 